Results Our data show that during fracture repair the majority of lymphocytes, macrophages and eosinophils appear late upon ensuing endochondral bone formation. Interestingly, lymphopenic mice had the capacity to mineralise cartilage and undergo subsequent resorption earlier than WT controls in vivo. Accordingly, by histomorphometric comparison of fracture repair in different immunodeficient animal models, the authors found that the speed of endochondral bone formation is $\mathrm{T}$ cell dependent. Confocal microscopy also showed that accelerated mineralisation in RAG mice occurred well before lymphocytes could be detected within the callus of WT animals. However, the authors clearly visualised in the callus of RAG and normal mice the concomitant immigration of osteogenic precursors from surrounding tissues. Remarkably, bone marrow osteogenic cells from RAG mice, taken in normal conditions, displayed abnormal mineralisation capacity than normal controls upon cell culture. This suggests that lymphocytes control bone regeneration by programming the basal activity and capacity to respond to damage of osteoblast precursors.

Conclusions Bone repair involves the migration of bone marrow osteogenic precursors into the bloodstream to reach fracture sites.

Immune cells infiltrate the fracture callus during the midlate stages of bone regeneration and they consist mainly of $B$ lymphocytes.

$T$ cells control the velocity of endochondral bone formation by programming the mineralisation capacity of osteogenic cells.

\title{
6 LYMPHOCYTES CONTROL BONE FRACTURE HEALING BY PROGRAMIMING THE MINERALISATION CAPACITY OF MIGRATORY OSTEOGENIC PRECURSORS
}

Alessandro Serra, ${ }^{1}$ Ireen Könnecke, ${ }^{2}$ Katharina Schmidt-Bleek, ${ }^{2}$ Hanna Schell, ${ }^{2}$ Andreas Radbruch, ${ }^{1}$ Georg Duda ${ }^{2}$ Deutsches Rheumaforschungszentrum Berlin, Berlin, Germany; 'Julius Wolff Institut, Charité, Universitätsmedizin Berlin, Berlin, Germany

10.1136/annrheumdis-2011-201237.6

Background and objectives Inflammation of the bone microenvironment in arthritic conditions leads to extensive infiltration of immune cells causing destruction of articular cartilage and bone. Similarities characterise bone fracture. Here, sustained inflammation leads to synthesis of bone and cartilage, massive infiltration of hematopoietic cells and controlled tissue resorption terminating into net bone formation. Thus, to better understand how bone inflammation leads to successful repair instead of autoimmunity, the authors have drawn a comprehensive cellular study of post-fracture bone regeneration by confocal microscopy and other histological techniques.

Materials and methods Experimental closed femoral fracture was used to induce bone regeneration in C57BL/ six mice and congenic RAG ${ }^{-1-} \mathrm{KO}$ (lymphopenic), TCR $\beta \delta$ chain $^{-/-} \mathrm{KO}$ ( $\mathrm{T}$ cell deficient) and $\mathrm{JHT}^{-/-} \mathrm{KO}$ (B cell deficient) mice. Confocal microscopy was used to detect the presence of T, B, macrophages, eosinophils, activated osteoclasts, osteogenic precursors and osteblasts in the fracture callus. Histomorphometry was used to quantify bone regeneration. Cell culture was used to measure mineralisation capacity at steady state of bone marrow derived osteogenic precursors in WT and lymphopenic mice. 